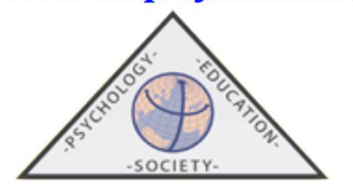

\title{
Investigación-acción en la enseñanza de la historia: simulación arqueológica en entornos educativos formales
}

\author{
Irene PALOMERO-ILARDIA
}

Universidad Rey Juan Carlos

(Recibido el 16 de Mayo de 2019, Aceptado el 29 de Septiembre de 2020)

RESUMEN: El currículo de Educación Primaria del sistema educativo español reserva el estudio de la Historia y nuestro pasado a los últimos cursos (a partir de los 8-9 años). Esto se debe, entre otros motivos, a las dificultades existentes en el aprendizaje de nociones temporales.

Historia y Arqueología son Ciencias Sociales que comparten elementos, pero tienen diferente utilidad didáctica. El uso de la Arqueología en el aula debe considerarse, no como actividad lúdica que "entretenga" a los alumnos, sino como una ciencia que pueda acompañar y combinar el estudio de las diversas técnicas de trabajo arqueológicas para comprender la materia curricular de Historia.

Para comprobar la utilidad de los contenidos de la Arqueología en beneficio de la Historia, se ha optado por seguir la metodología de la investigación-acción, que nos permite establecer un diagnóstico, elaborar un plan de acción que sea puesto en práctica y reflexionar sobre los resultados obtenidos.

A partir de una experiencia con alumnos de $4^{\circ}$ de Primaria de un centro educativo español, se analiza una actividad que busca, no solo entretener o sacar de la rutina a los educandos, sino que pretende conseguir el desarrollo de determinadas competencias y el aprendizaje de contenidos diversos.

Palabras clave (3-5): excavación arqueológica simulada; educación primaria; didáctica de la Historia; pasado, educación formal.

\section{Action Research in Teaching History: Archeological Simulation in Formal Educational Settings}

\begin{abstract}
The Primary Education curriculum of the Spanish educational system reserves the study of History and our past to the last years: normally it is in the third year (8-9 years) when the study begins. This is due, among other reasons, to the difficulties in learning temporal notions.

History and Archeology are Social Sciences that share elements, but have different didactic purposes. The use of Archeology in the classroom should be considered, not as a playful activity that "entertains" the students, but as a science that can accompany and combine the study of the various archaeological work techniques to understand the curriculum of History.-
\end{abstract}


In order to verify the usefulness of the contents of Archeology for the benefit of History, it has been chosen to follow the action research methodology, which allows us to establish a diagnosis, prepare an action plan that is put into practice and reflect on the results obtained.

Based on an experience with 4th grade students from a Spanish educational center, we make the analysis of an activity that seeks, not only to entertain or remove students from routine, but also to achieve the development of certain competences and content learning diverse.

Keywords: Simulated Archaeological Excavation; Primary Education; Didactics on History; Past; Formal Education.

Correspondencia: Irene Palomero Ilardia. C/ Ana María, no 32, 1 C. 28039 Madrid. E-mail: irene.palomero@urjc.es

\section{Introducción}

En el sistema educativo español, el pasado se comienza a trabajar durante el segundo ciclo de Educación Infantil (3 a 6 años), con el aprendizaje de conceptos relacionados con el tiempo, incluyendo en ocasiones culturas o civilizaciones antiguas. No es hasta la etapa de Educación Primaria (6 a 12 años), cuando, avanzando hacia un conocimiento más profundo y abstracto del tiempo y su forma de medirlo, se puede comenzar el estudio de temas relacionados con la Historia y nuestro pasado.

Para llegar hasta ese nivel de comprensión, la mente del educando ha pasado por unas fases previas que han ido conformando las ideas de tiempo, paso del tiempo; aprehender los conceptos de pasado-presente-futuro, rutina-costumbre-tradición, día-semana-mes-año (Pagés y Santiesteban, 2010). Por lo tanto, la interiorización de la noción del tiempo está directamente relacionada con el aprendizaje de la Historia y, si no tenemos unos mimbres temporales bien tejidos y entrelazados, no se podrá crear el "cesto histórico" donde guardar los frutos a modo de etapas históricas. El único inconveniente sería que, para lograrlo, haría falta un nivel de abstracción mental que no se alcanza casi hasta la adolescencia, por lo que no parece posible trabajarlo en el nivel educativo de primaria... ¿O quizás sí es posible?

Este texto pretende contestar afirmativamente a esta cuestión, dando una oportunidad al conocimiento de la Historia a través de otra ciencia social más reciente en su conformación: La Arqueología. Historia y Arqueología son ciencias sociales que comparten elementos, pero tienen diferente utilidad didáctica (Santacana y Hernández, 1999). La Arqueología en el aula debe de ser vista, por docentes y educandos, no como una actividad lúdica que "entretenga" a los alumnos, sino como una ciencia que pueda acompañar y combinar el estudio de las diversas técnicas de trabajo arqueológicas con la comprensión de la materia curricular de Historia.

Por tanto, en primer lugar, deberemos analizar la utilidad de la Arqueología en entornos educativos formales para conseguir el aprendizaje de la Historia, y posteriormente, pondremos en práctica la metodología de investigación en la acción (Lewin, 1973, Stenhouse, 1984 y 1987, Elliot, 1990 y 1993) para comprobar su eficacia como elemento transformador del proceso de aprendizaje. 


\section{Utilidad de la arqueología en entornos educativos formales}

Sin querer entrar a profundizar demasiado en las teorías, dado que no es el objeto de este artículo, sí podemos mencionar algunas de las fuentes en las que nos inspiramos para justificar teórica o metodológicamente la utilidad de una ciencia como la Arqueología en el entorno escolar.

Siempre se ha considerado, por parte de aquéllos que pretenden renovar la enseñanza, que la Historia es un conjunto de datos, fechas, nombres, que han de ser memorizados para posteriormente ser olvidados. Es cierto que, en muchas ocasiones, sentimos que recibimos gran cantidad de información que olvidamos tan rápido, sin llegar siquiera a crear un vínculo con la misma. Asimismo, aún en la actualidad, subsiste en el inconsciente colectivo la idea de que la Historia, el estudio del pasado o de otras civilizaciones, es algo tedioso, aburrido y que aporta pocas cosas útiles para la vida real. Si bien este tipo de pensamientos han de ser desterrados por inciertos a todas luces, también es verdad que hay que intentar innovar para borrar esos pensamientos de nuestra sociedad.

Para intentar paliar esta falta de motivación, en los últimos años se han desarrollado novedosas investigaciones sobre la neurodidáctica, llegando a afirmar que educar es modificar al cerebro o que todo educador es modificador del cerebro (Paniagua, 2013); que la motivación es un proceso interno que, gracias a la dopamina, nos permite mantener la atención y potenciar la memoria (Gruber et ali, 2014); que la motivación del alumno depende de lo atractiva e interesante que sea la situación del aprendizaje (Cabanach, 1996, citado por García Bacete, y Doménech Betoret,1997), o que la información buscada que parte del interés y la curiosidad es la que facilita el aprendizaje.

La idea de conectar los conocimientos nuevos con los ya existentes en nuestro cerebro ayuda a retener la información nueva, porque ésta se vincula y conecta físicamente dentro de una red de información, de tal modo que la información que se integra en una red existente se activará cada vez que se active la red (Stamm, 2018). Esto nos lleva a pensar en el aprendizaje significativo.

Y el hecho de incorporar diversión y entusiasmo en las clases para fomentar la atención y que ésta fomente la memoria (Stamm, 2018), es también una idea que parte de la neurociencia aplicada a la educación.

Si damos por ciertas estas afirmaciones, no podemos sino concluir que, en el ámbito de la Historia, cuanta mayor motivación, interés y curiosidad despertemos en los alumnos sobre el pasado, mejor será el aprendizaje del mismo. Si trabajamos el aprendizaje significativo y logramos transmitir entusiasmo, conseguiremos fomentar la atención y, por tanto, la memoria, tan necesaria en una materia como es la Historia.

Por lo que respecta a la Arqueología, ciencia social que estudia el pasado del ser humano y sus restos materiales, puede que la motivación, el interés y la curiosidad sean mayores que en la Historia: Ya sea por lo atractivo de descubrir algo oculto durante mucho tiempo, por tratarse de una actividad que requiere un trabajo manual en su realización y un contacto con herramientas no usuales y objetos, o incluso por la expectación -y la imagen totalmente desvirtuada tambiéncreada por la industria del cine durante demasiado tiempo, la motivación es una realidad.

La actividad arqueológica puede suponer también el contacto con objetos que, ya sean de un pasado muy lejano o más cercano, dan a los arqueólogos informaciones muy diversas sobre 
la posible forma de vida, la economía, la organización social, las creencias religiosas y otros elementos de una sociedad pasada. El trabajo con objetos podría incluirse dentro del concepto de aprendizaje significativo (Ausubel, 1973, 1976, 2002 y Rodríguez-Palmero, 2004, 2011) en el sentido de que podemos asociarlos con fechas, personas, construcciones, civilizaciones, imágenes o representaciones mentales culturales de cada época.

Asimismo, las experiencias manipulativas en niños de edades infantiles son fundamentales para su aprendizaje. El sentido del tacto ayuda en los primeros años a formar conceptos explorando las sensaciones que despierta lo que nos rodea (Stamm, 2018). La actividad arqueológica, a diferencia de la Historia, tiene un carácter manipulativo, experimental y, al mismo tiempo, intelectual (Bardavio y Mañé, 2017) que permite un aprendizaje no estático y meramente receptivo, sino participativo, que potencia el aprendizaje significativo, que los acerca al hecho histórico rompiendo las barreras existentes con el libro de texto y la simple recepción de conocimientos cerrados e inamovibles.

Por otro lado, si analizamos cuál es el procedimiento de trabajo de los arqueólogos, su metodología puede equipararse al aprendizaje basado en problemas (ABP). Los arqueólogos, al igual que los historiadores, se cuestionan las cosas, realizan preguntas y sus respuestas son las que les hacen avanzar en su trabajo. De este modo, el conocimiento se adquiere de la misma manera que un detective resuelve un caso: realizando las preguntas correctas y usando para ello sistemas de pensamiento deductivo y/o inductivo. En cierta manera, en esto consiste el método de investigación arqueológica, que además nos permite volver a las cuestiones iniciales una y otra vez. Si extrapolamos esta última idea a entornos educativos, también estaríamos trabajando el currículo en espiral.

En este punto debemos matizar que la pretensión de esta propuesta no es convertir a los estudiantes en arqueólogos, sino aplicar algunas de las metodologías de su proceso investigador en el aula, que pueden resultar beneficiosas para motivar, cambiar la visión del estudio del pasado, mejorar su aprendizaje, etc.

\section{Metodología: investigación-acción}

La metodología de Investigación-acción fue acunada y desarrollada inicialmente por Kurt Lewin (1947, citado por Elliot, 1990), quien entiende la enseñanza como un proceso de investigación y continua búsqueda. En el desarrollo de esta idea, aparecen otros autores que lo entienden como "el estudio de una situación social para tratar de mejorar la calidad de la educación en la misma" (Elliot, 1993) y como un tipo de investigación en la que "el acto investigador es necesariamente un acto sustantivo, el acto de averiguar tiene que ser acometido con una obligación de beneficiar a otros que pertenecen a la comunidad" (Stenhouse, 1987).

La decisión de usar esta metodología se basa, además de en algunos de los principios establecidos por estos autores, en la idea de que la educación puede mejorarse a través del cambio, aprendiendo a partir de las consecuencias que provocan esos cambios (Kemmis y McTaggart, 1988).

Siguiendo el proceso o ciclo establecido (Kemmis y McTaggart, 1988), la metodología empleada en el estudio de caso que presentamos comenzará el ciclo con un diagnóstico o reconocimiento de la situación inicial, para establecer un plan de acción, que será observado y analizado 
en el contexto en el que se realice. Finalmente, habrá una fase de reflexión y evaluación sobre los efectos, que será la base de una nueva planificación, cerrándose así un ciclo y abriéndose otro en forma de espiral.

Dentro de las tipologías de investigación-acción que se han ido definiendo en base a diferentes criterios (Carr y Kemmis, 1988, Desroche, 1981 y Grundy, 1982), optamos por el modelo "práctico" propuesto por Grundy, que abarca los procesos de investigación-acción dirigidos a la realización de aquellos valores intrínsecos a la práctica educativa, que implica una indagación y reflexión de la práctica a la luz de sus fines y viceversa, de los fines o valores a la luz de los acontecimientos prácticos (Bausuela, 2004). Esta modalidad coincide asimismo con los planeamientos de Stenhouse y Elliot.

A continuación, iremos viendo cada una de las fases de este ciclo aplicadas a una propuesta concreta, para introducir ciertas metodologías de investigación provenientes de la Arqueología, que pensamos pueden contribuir de forma determinante en el mejor aprendizaje y comprensión de la Historia como materia curricular.

\section{Diagnóstico en la investigación-acción en el contexto educativo formal}

Partimos de varias hipótesis en el reconocimiento de una situación inicial, en base a diferentes entrevistas y charlas informales mantenidas con docentes de los niveles educativos que van a desarrollar las actividades en el aula. Tras realizarlas, parece que hay una serie de ideas que destacan: La Historia no es atractiva en el ámbito educativo formal y el sistema de enseñanza tradicional de la Historia no favorece la fijación del conocimiento ni la aprehensión de conceptos básicos del currículo. Otra de las ideas a destacar es que a la Historia le falta un elemento práctico y tangible que permita a los alumnos "engancharse" y motivarse.

Asimismo, de estas charlas informales se obtuvo información sobre el interés que los docentes tenían en realizar algún tipo de actividad innovadora en las instalaciones educativas en relación con los contenidos de Historia que recientemente se habían trabajado, si bien la falta de formación sobre otras metodologías y la falta de tiempo para poder investigar sobre la mejor manera de plantearlo eran algunos de los condicionantes más destacables.

Por tanto, el diagnóstico que se podría establecer es que, ante la problemática de falta de atractivo y la ausencia de elementos tangibles que vinculen a los educandos con la Historia, se hace necesario encontrar una herramienta con cierto grado de innovación con la que paliar las dificultades que entraña el aprendizaje del pasado en general y de la Historia en particular, que sea fácilmente realizable en el entorno del centro educativo.

Intentando aunar todas estas ideas, podemos establecer en nuestra metodología de investigación-acción que, en base a este diagnóstico, se pretende conseguir una herramienta que favorezca el adquirir los conocimientos históricos, establecidos en el currículo, mediante elementos

prácticos y tangibles, consiguiendo además una mayor involucración en el aprendizaje de los alumnos y una innovación con respecto a la manera tradicional de enfocar el estudio de esta materia.

En base a estas ideas, se procede a elaborar un plan de acción que intente mejorar la situación actual, tomando como punto de partida los elementos prácticos que nos pueda aportar la Arqueología. 


\section{Plan de acción: ¿cómo se puede introducir la arqueología en el aula?}

Una vez analizadas las virtudes del trabajo arqueológico, que pueden aplicarse en un entorno educativo formal, la siguiente cuestión que nos planteamos es de qué modo enfocar esta materia y cómo exportar algunas de las metodologías arqueológicas al aula, a través de la simulación. Podemos valorar dos grandes formas de acercarnos al pasado y al trabajo arqueológico.

En primer lugar, realizar talleres en centros externos al aula, ya sea tras la visita a un museo, un yacimiento o como actividad independiente. Actualmente contamos con variados ejemplos en nuestro país, bien en museos, o bien vinculados a enclaves arqueológicos; pero también existen otros espacios pensados en exclusiva para los más pequeños, como el Parque de la Prehistoria de Teverga (Suárez, 2011), Arqueopinto, Camp d'aprenentatge de la Noguera (Bardavio y González, 2008, Bardavio y Mañé, 2018), CAREX ...Todos ellos, no siendo clasificables en una única tipología, tienen en común que realizan talleres variados y adaptados a la edad, con expertos en didáctica, materiales de buena calidad y espacios adecuados. En algunas ocasiones, además, se pueden combinar los talleres con la visita a un yacimiento arqueológico -como es el caso de Altamira o de Atapuerca-, o a un museo arqueológico (Museo Arqueológico Nacional, Museo de Arqueología de Alicante). Los departamentos de difusión, educación y didáctica elaboran su propia programación de actividades a lo largo del año, que suelen estar publicadas en la web de cada institución. Entre ellos, destaca la simulación de excavación arqueológica.

Estos talleres, siendo una opción muy buena y recomendable, tienen la desventaja de que pueden romper el horario lectivo, suponen un desplazamiento del centro escolar en ocasiones no posible, no siempre se adaptan a la edad de los alumnos o se centran en un período histórico concreto. Estos inconvenientes se pueden paliar realizando el taller en el colegio, ya que podemos crear una actividad "a la carta", es decir, adaptada a las necesidades del aula, a la edad de los alumnos y a lo que nos interese trabajar en cada situación.

Esta segunda opción, objeto de análisis y base de nuestro plan de acción, consiste en realizar una simulación de excavación arqueológica en el aula o en el patio de la escuela. Se llevan al colegio muchos de los elementos que se trabajan en los talleres de museos y yacimientos, añadiendo otros que sería más complicado trabajar fuera del aula. Este tipo de actividad es totalmente factible en la actualidad en un centro educativo gracias a los diversos materiales didácticos disponibles para los docentes: maletas didácticas, réplicas, arqueología experimental, cajones de excavación, etc.

Por otro lado, la realización de esta actividad en el centro educativo nos permite seleccionar los objetos y así acomodar los contenidos que queramos trabajar. Con niños de la etapa infantil podríamos trabajar las nociones básicas del tiempo cronológico, del pasado-presente; o bien clasificar los objetos del pasado y el presente, sin necesidad de usar una cronología ni ubicarlos en una civilización o período histórico concreto. Los conceptos "antiguo/moderno" también podrían introducirse para realizar la clasificación de los objetos, o bien el tipo de material con el que están hechos o el uso que se les dio en el pasado. En los primeros cursos de la etapa de Educación Primaria, podremos añadir a estos contenidos la asociación de los mismos con una cultura o civilización, e incluso a un período histórico amplio (Prehistoria, Edad Antigua, etc). Y, a partir de los 10-11 años, ya podríamos incorporar la cronología básica que hayan trabajado, 
escogiendo objetos que se puedan asociar con períodos históricos, si bien la dinámica de saber identificar materiales antiguos y nuevos también puede funcionar en estas edades.

Además de estos conceptos, la simulación de excavación arqueológica nos permite introducir algunos de los elementos del método científico con el que trabajan los arqueólogos: documentación gráfica y escrita, estudio posterior de los materiales y clasificación en laboratorio; "preguntar a los objetos". Dependiendo de la edad con la que se trabaje, esta parte variará en su complejidad o en la manera de llevarlo a la práctica.

Otro de los contenidos que se pueden desarrollar con este tipo de taller es aprender cuál es el verdadero trabajo de los arqueólogos. A pesar de lo que transmitan producciones cinematográficas infantiles, es importante dejar claro que, en el entorno escolar, lo interesante es aprender la labor científica e investigadora que realizan habitualmente. Imitando algunas de las funciones que desempeñan (excavar un yacimiento), viendo que el tipo de herramientas que utilizan para ello son más parecidos a las observables en una obra (pala, cepillo, carretilla, paletines) que a las de un aventurero, es sencillo comprender la diferencia existente.

Como ya hemos comentado, esta actividad tiene un eje principal: el trabajo con objetos. Dichos objetos no serán originales sino réplicas o elaboraciones propias que los imiten, lo que permitirá a los educandos "tocar el pasado", aprender el pasado a través de los objetos que lo representan.

Por otro lado, el trabajo con objetos previamente encontrados por los alumnos nos abre un gran abanico de posibilidades en el trabajo conceptual, procedimental y actitudinal (Morales y Egea, 2018). Por un lado, nos ayuda a explicar la cultura material de la sociedad que los utilizó, sus usos y costumbres. De este modo, la Historia dejará de ser un listado de las características de dicha sociedad, y, gracias a la Arqueología, nos acercaremos un poco más a la Historia "real" de la época estudiada. Asociar los objetos que se hayan localizado en la excavación simulada con la vida cotidiana de sociedades anteriores, las necesidades, la economía, los rituales, el ornamento, y la comprensión de la actividad humana a lo largo del tiempo puede ser muy útil para el aprendizaje.

También podemos realizar una labor de comparación entre objetos cotidianos del pasado y del presente, e incluso introducir el trabajo de los restauradores y conservadores -en el caso de trabajar con piezas fragmentadas-, o un responsable de museo, si concluimos la actividad de simulación de excavación con la creación de un museo donde se exhiban las piezas localizadas. Y podríamos enlazar todo esto con un taller de elaboración de objetos con técnicas tradicionales, introduciendo de este modo la arqueología experimental en el aula.

Por tanto, las opciones son muy variadas y, combinándolas para buscar distintos objetivos, permiten trabajar los conceptos que sean de nuestro interés y adaptarse a las necesidades del grupo en cada momento. La actividad puede incluso plantearse en diferentes momentos del calendario escolar, ya sea previa al comienzo del estudio de la materia, intermedia o de cierre. La elección del momento dependerá de lo que pretendamos lograr con la misma: una motivación o aliciente, una forma de ver la evolución en el aprendizaje, o de evaluar los conocimientos, el trabajo en equipo, etc.

Llegados a este punto, se hace necesario diferenciar los objetivos de la propuesta didáctica (taller) de los propios del proceso de investigación-acción llevado a cabo.

En lo relativo al taller, los objetivos perseguidos desde el punto de vista didáctico, son: 
- Poner en práctica algunos contenidos estudiados previamente en la materia de Historia.

- Encontrar, reconocer y clasificar los objetos en función de su antigüedad o el material con el que están elaborados, asociándolos a una etapa histórica y con una cronología clara.

- Simular el trabajo de un arqueólogo e introducir el método científico.

Por otro lado, nuestro plan de acción se basa en la creación de un taller de excavación simulada, cuyo objetivo principal es que sirva a los docentes para poder tomar decisiones didácticas sobre la utilidad de la metodología de investigación arqueológica, como complemento ideal en el aprendizaje de contenidos históricos básicos, pero también procedimentales y actitudinales, y sobre el trabajo colaborativo de los alumnos. Desgranando un poco estas ideas, podemos marcar como objetivos del plan de acción:

- Encontrar elementos tangibles que provoquen una vinculación de alumnos con Historia.

- Disminuir las dificultades en el aprendizaje del pasado en general.

- Desarrollar una actividad innovadora y fácilmente implementable en el aula.

\section{Acción: experiencia real en el aula y observación}

A modo de contextualización, se explica a continuación la experiencia real implementada en dos grupos de $4^{\circ}$ curso de Educación Primaria en un colegio público de la localidad de Picassent (Valencia). Se trata de una actividad de cierre realizada en dos sesiones, una por grupo, de aproximadamente hora y media cada una.

La actividad propuesta buscaba, desde el punto de vista de la metodología de investigación- acción, crear un complemento totalmente práctico de los contenidos teóricos ya estudiados, usando elementos tangibles y permitiendo una mayor motivación e involucración de los estudiantes en su realización; $y$, desde el punto de vista didáctico, asentar contenidos conceptuales básicos, poner en práctica contenidos procedimentales, trabajar algunos actitudinales y desarrollar ciertas competencias: valorar los restos materiales del pasado, como fuentes de conocimiento y aprendizaje; conocer el uso y el proceso de fabricación de las herramientas; ampliar la curiosidad y el afán por aprender sobre la arqueología y las primeras etapas históricas; conocer y respetar las formas de vida y costumbres de los antepasados; proporcionar a los alumnos/as un contacto directo con su pasado para ayudarles a entender mejor su presente y de ese modo consolidar su identidad individual y colectiva; desarrollar técnicas de investigación, el aprendizaje por descubrimiento y fomentar la competencia de aprender a aprender.

El equipo docente decidió realizar el taller dentro del aula, donde se colocó una caja de plástico transparente, no muy profunda y de dimensiones suficientes para subdividir el espacio de trabajo en sectores de actividad, y permitir excavar a varios alumnos a la vez (Figura 1). 


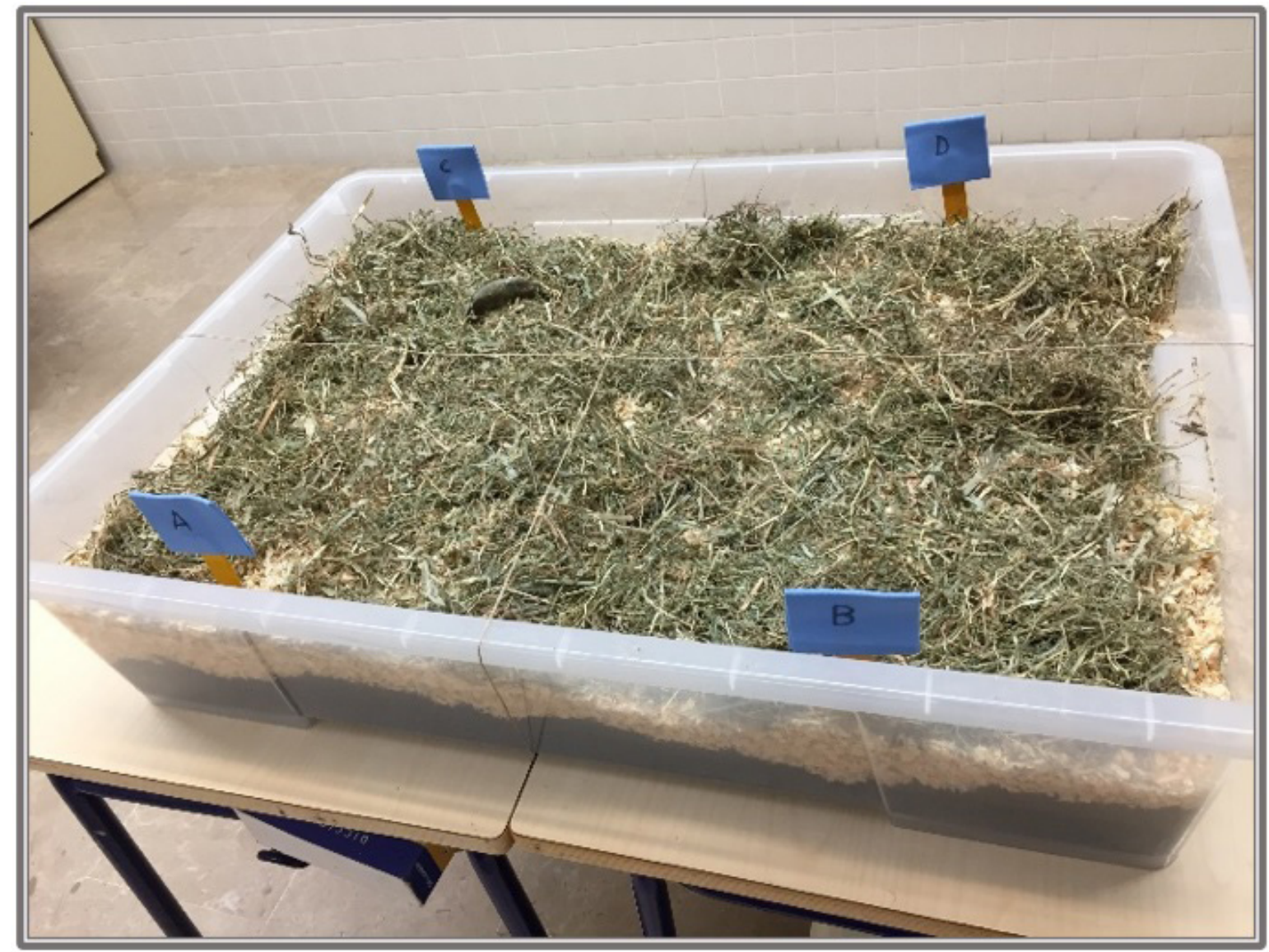

Figura 1. Caja de excavación antes de comenzar la actividad.

Antes de comenzar la actividad, se dividió a los alumnos por grupos, cada uno en un área de excavación, y a cada uno de sus miembros se le asignó una tarea. Se les explicó cómo extraer la tierra y los objetos que fueran localizando, así como los pasos a seguir a continuación (dibujo del objeto, limpieza, elaboración de ficha, exposición).

Una vez localizados todos los objetos de su sector, el grupo debía limpiarlos, asignarles un código alfanumérico y meterlos en una bolsa transparente, rellenar una ficha de uno de ellos y cartelas del resto para su posterior exhibición. (Figura 2). Para completar algunos de sus apartados, los alumnos tenían que aplicar los conocimientos previos de Historia que ya tenían (descripción del objeto, cronología, período, material); sin embargo, para otros (medidas, color, dibujo) utilizaron la observación y el manejo de instrumental adecuado (pie de rey, reglas, pinceles). 

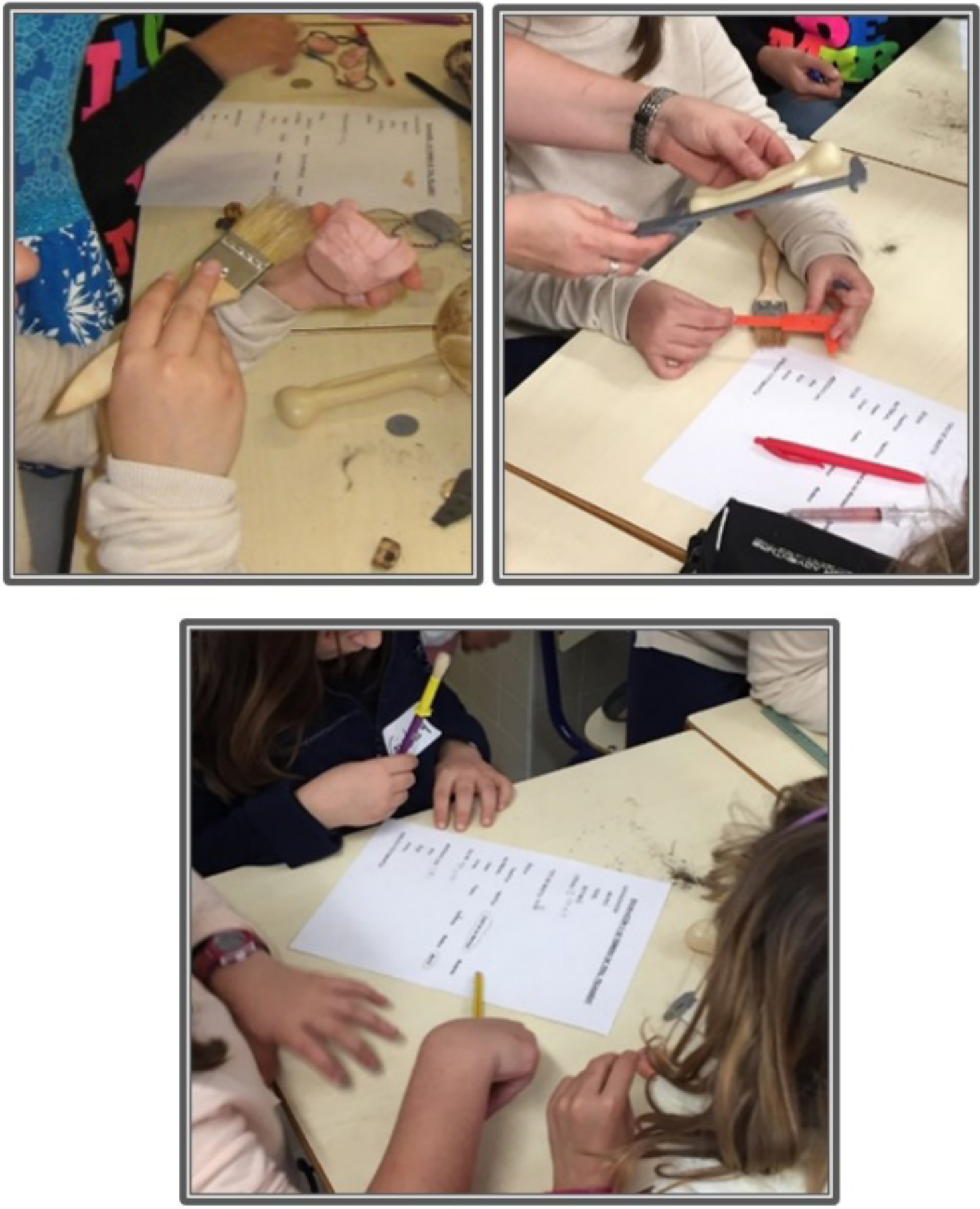

Figura 2. Proceso de trabajo tras la excavación.

Una vez realizado este trabajo, cada grupo debía crear su sala del museo donde exponer todos los objetos encontrados a modo de vitrina, aportando la información básica de cada uno de ellos (objeto, material, período), mediante una cartela (Figura 3). Asimismo, tenían que ser capaces de explicar esa información a los compañeros de los otros grupos. 


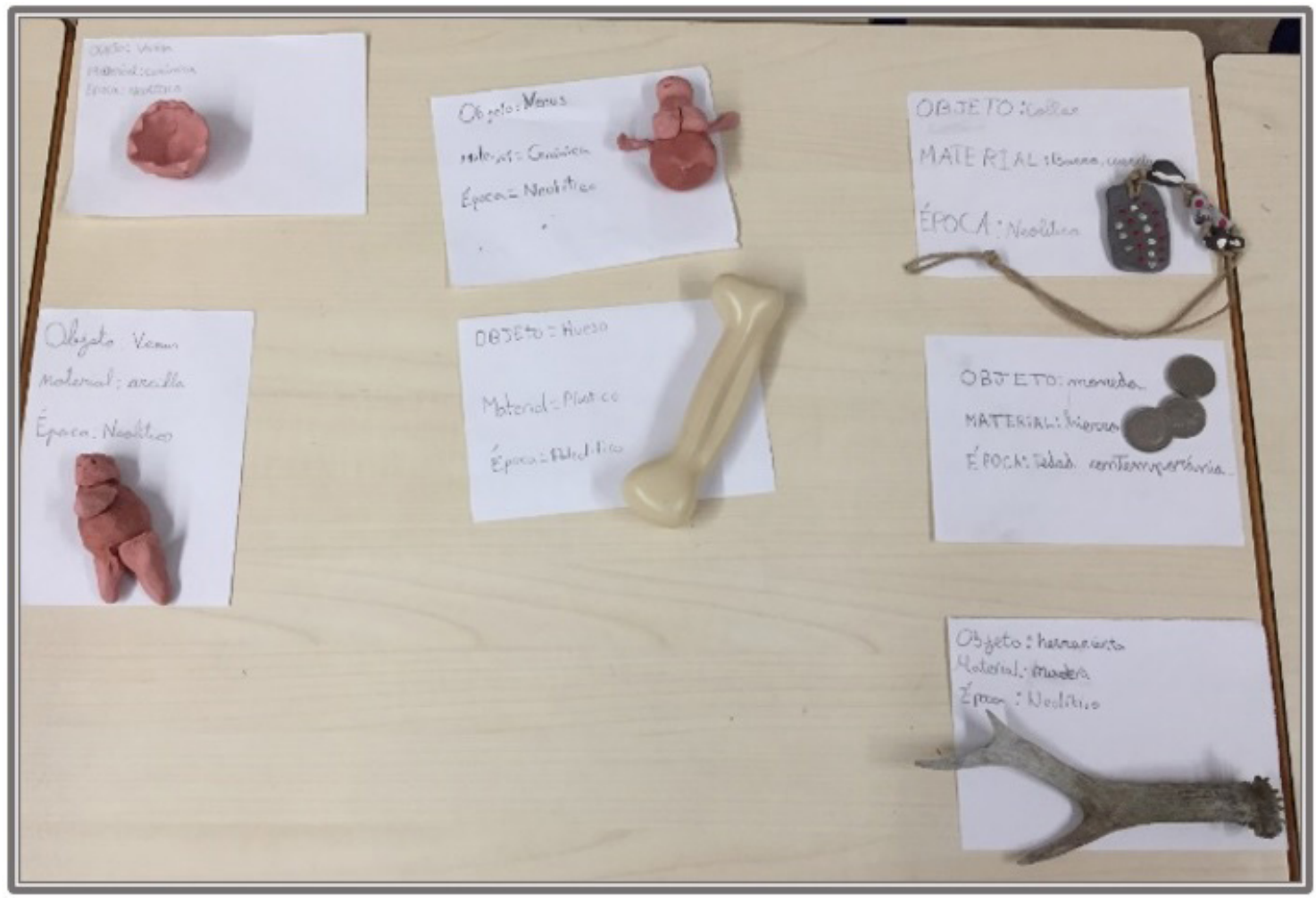

Figura 3. Objetos y cartelas realizadas para la exposición (museo).

Durante el desarrollo del taller, se llevó a cabo un análisis mediante la observación, tomando nota de las impresiones y reacciones de los alumnos. En un primer momento, los alumnos mostraron un gran interés por la acción concreta de desenterrar cuantos más objetos mejor, como si estuvieran buscando un tesoro, sin tener en cuenta los objetos localizados en sí mismos ni el hecho de que debían elaborar una ficha con la información básica de cada uno.

Finalizada la excavación, se fue haciendo una ronda por todos los grupos, observando cómo clasificaban y elaboraban las fichas y cartelas. Se pudo comprobar, mediante diferentes preguntas, que el aprendizaje previo de ciertos contenidos teóricos había sido fundamental para reconocer y situar cronológicamente los objetos, pero que el poder identificar visualmente y sobre todo tocar un objeto representativo de ese período, facilitaba al alumno el vínculo del mismo con un período histórico.

Las docentes pudieron observar cómo el trabajo colaborativo y los debates que generaba cada uno de los objetos entre los compañeros de grupo, contribuían a que aquellos alumnos con dificultades previas en esta materia y que tenían dudas en la identificación del período al que podrían corresponder o la cronología aproximada, las resolvieran y afianzaran estos conocimientos. Asimismo, el trabajo colaborativo y la cooperación entre los alumnos se desveló como fundamental para la solución de los problemas.

Por último, en el proceso de elaboración de las fichas y cartelas, los alumnos tuvieron que hacer preguntas a los objetos (como el material del que estaban hechos, su función, o si se había localizado en la parte superior o inferior del cajón de excavación) y algunas de las respuestas 
obtenidas les permitían, aplicando los conocimientos aprendidos de cada etapa histórica, encajar cada objeto en una cronología concreta y acertada.

\section{Conclusiones: Fase de Reflexión y evaluación}

El proceso de Investigación-acción establece que la última fase del ciclo sea la reflexión y evaluación de los efectos del plan de acción, que en nuestro caso va a conformar el apartado de conclusiones en cierto modo.

En cuanto al desarrollo de la actividad, tanto el alumnado como el personal docente la valoró como interesante, entretenida e innovadora, si bien aportaremos algunas reflexiones más concretas que nos han surgido tras analizarla en profundidad y que enfocan su visión hacia una mejora de la actividad en sí misma:

- El trabajo durante la actividad, para docentes y educandos, debe ser muy metodológico. Es necesario que el docente explique previamente las diferentes funciones que van a realizar, estableciendo un orden en las fases que han de seguirse, pero es igualmente importante que los educandos sigan esas pautas lo más fielmente posible, para lograr un trabajo eficaz con resultados positivos.

- Los grupos han de ser lo más reducidos que sea posible. Cuantos menos educandos realicen la actividad simultáneamente, la atención podrá ser más personalizada e incluso el docente podría usarla como un elemento evaluador.

- Si nuestra intención es que la actividad sea vista como un complemento del aprendizaje y no exclusivamente como algo entretenido, se debe de aprovechar el aprendizaje significativo para lograr que los alumnos interconecten los conocimientos teóricos ya aprendidos con los objetos que están representando a cada período, realizando reflexiones. Poner en práctica el saber hacer o saber interpretar y saber explicar son fundamentales en este tipo de talleres y en este caso, tratándose de una actividad de cierre, ese era uno de los objetivos. De este modo, sería recomendable que, mientras los alumnos realizan las fichas, se haga un trabajo de reflexión con ellos, ayudándoles en el proceso de investigación, formulando las preguntas adecuadas y guiando el aprendizaje.

- Es muy importante aportar a los alumnos una ficha de trabajo para centrar el tipo de información que deben obtener y ordenar los pasos que han de seguir en su desarrollo. Hay que intentar que el hecho concreto de excavar y encontrar un objeto, no se quede simplemente en eso y que los alumnos tengan claro que el fin último de la excavación va más allá del acto concreto de localizar piezas antiguas de valor, sino que el objeto debe servirnos para aportar alguna información relevante.

- Hubo dificultades en la identificación de algunos objetos antiguos frente a otros, no tanto en relación con el material del que estaban hechos, sino en cuanto al reconocimiento del tipo de objeto, a su denominación y a su posible función. Esto pudo deberse a que muchos de los objetos del pasado estaban fragmentados y no se podían ver completos salvo que se restaurase la pieza, cosa que no se pudo realizar por falta de tiempo.

- Se fomenta el trabajo colaborativo, la investigación en grupo, la reflexión y el trabajo de valores. Aprender a trabajar en equipo, a tomar decisiones buscando el consenso son valores que pudieron trabajarse con esta actividad, así como comprender cuál es la la- 
bor real de los profesionales de la Arqueología y aprender que el esfuerzo de un trabajo de investigación da sus frutos.

- La motivación y entusiasmo despertados en el conjunto del alumnado fue tremendamente positiva y satisfactoria.

En relación con el diagnóstico propuesto al comienzo de este artículo - encontrar una herramienta que favorezca el adquirir conocimientos históricos establecidos en el currículo, mediante elementos prácticos y tangibles, consiguiendo además una mayor involucración en el aprendizaje de los alumnos-, podemos afirmar, en primer lugar, que todos los alumnos que realizaron el taller participaron de forma activa, ya fuera por lo novedoso, por el contenido eminentemente práctico que tenía, por el trabajo en grupo que requería, o por la posibilidad de manipular e investigar a través de objetos.

Por tanto, en este proceso de reflexión y evaluación del taller de simulación de excavación, con las mejoras que se han indicado con anterioridad, podemos concluir que se ha mejorado la educación mediante un cambio y se ha aprendido también de las consecuencias de los cambios (Kemmis y McTaggart, 1988), se ha mejorado la calidad de la educación (Elliot, 1993). Asimismo, el acto investigador llevado a cabo ha sido también un acto sustantivo (Stenhouse, 1987), puesto que el taller realizado sirvió para ayudar en el aprendizaje de los alumnos (acto sustantivo), a la vez que una herramienta que nos ha servido para realizar la investigación (acto investigador).

Por tanto, como afirman los autores de la metodología de investigación-acción, se ha podido llevar a cabo una transformación social y podemos considerar que este tipo de actividad supone una mejora en el aprendizaje de contenidos históricos en un ámbito educativo formal y desde una perspectiva más práctica que teórica.

\section{Referencias}

Ausubel, D. P. (1973). Algunos aspectos psicológicos de la estructura del conocimiento. En Elam, S. (Comp.). La educación y la estructura del conocimiento. Investigaciones sobre el proceso de aprendizaje y la naturaleza de las disciplinas que integran el currículum (pp.211-239). Buenos Aires: El Ateneo.

Ausubel, D. P. (1976). Psicología educativa. Un punto de vista cognoscitivo. México: Trillas.

Ausubel, D. P. (2002). Adquisición y retención del conocimiento. Una perspectiva cognitiva. Barcelona: Paidós.

Bardavio, A. y González, P. (2008) El Campo de Aprendizaje de la Noguera: un proyecto integrado de arqueología experimental. Revista Iber Didáctica de las Ciencias Sociales, Geografía e Historia 57, $25-38$

Bardavio, A. y Mañé, S. (2017) La arqueología en la enseñanza obligatoria. El ejemplo del Campo de aprendizaje de la Noguera. En Reyes, F. Polo, A y Palomero, I. (Eds) Actas del Congreso COTARQ, Revista Otarq, 2, (pp.331-345), Madrid.

Bardavio, A. y Mañé, S. (2018). El campo de aprendizaje de La Noguera. Formar desde la arqueología y el patrimonio de la prehistoria en la educación pública. En Egea, A., Arias, L. y Santacana, J. (coords), Y la arqueología llegó al aula. La cultura material y el método arqueológico para la enseñanza de la historia y el patrimonio (pp. 291-324). Asturias: Ediciones Trea. 
Bausuela, E. (2004). La docencia a través de la investigación-acción. Revista Iberoamericana de Educación, 35, (1), 1-9. https://doi.org/10.35362/rie3512871

Campos, A. (2010). Neuroeducación: uniendo las neurociencias y la educación en la búsqueda del desarrollo humano. La Educación. Revista Digital, 143, 1-14.

Carr, W. y Kemmis, S (1988). Teoría crítica de la enseñanza. La investigación-acción en la formación del profesorado. Barcelona: Martínez Roca.

Desroche, H (1981). La recherche coopérative comme recherche-action. En Actes u Colloque recherte-action, Chicoutimi, UQAC, (pp. 9-48), octubre 1981.

Egea, A., Arias, L. y Santacana, J. (2018). Y la arqueología llegó al aula. La cultura material y el método arqueológico para la enseñanza de la historia y el patrimonio. Asturias: Ediciones Trea.

Elliot, J. (1990). La investigación-acción en educación. Madrid: Morata.

Elliot, J. (1993). El cambio educativo desde la investigación-acción. Madrid: Morata.

Forés, A. y Ligioz, M. (2009). Descubrir la neurodidáctica: Aprender desde, en y para la vida. España: UOC.

Forés, A., Gamo, J.R., Guillén, J.C., Hernández, T., Ligioiz, M., Pardo, F., Trinidad, C. (2015). Neuromitos en educación: el aprendizaje desde la neurociencia. Barcelona: Plataforma editorial.

García, F. J, Doménech, F. (1997). Motivación, aprendizaje y rendimiento escolar. Revista Electrónica de Motivación y emoción. 1 (0). Noviembre, 2-18.

http://reme.uji.es/articulos/pa0001/texto.html

Gardner, H. (1995). Inteligencias múltiples. La teoría en la práctica. Barcelona: Paidós.

Gruber, M., Gelman, B. y Ranganath, C. (2014). States of curiosity modulate hippocampusdependent learning via the dopaminergic circuit. Neuron, 84 (2). 486-496.

Grundy, S. (1982). Three modes of action research. En Kemmis, S y McTaggart, R. (ed.) The Action Research Reader ( ${ }^{\mathrm{a}}$ ed.), (pp. 354-364), Victoria: Deakin University

Kemmis, S. y McTaggart, R. (1988). Cómo planificar la investigación-acción. Barcelona: Laertes

Lewin, K (1973). Action research abd minority problems. En K. Lewin: Resolving Social Conflicts: Selected Papers on Group Dynamics (ed. G. Lewin) (pp.201-216). London: Souvenir Press.

Morales, M. J. y Egea, A. (2018). El uso de los objetos arqueológicos en las aulas de educación primaria. Diseño y análisis de una propuesta para un aula de ocho- nueve años. En Egea, A., Arias, L. y Santacana, J. (coords), Y la arqueología llegó al aula. La cultura material y el método arqueológico para la enseñanza de la historia y el patrimonio (pp. 137-158). Asturias: Ediciones Trea.

Pagès, J. y Santiesteban, A. (2010). La enseñanza y el aprendizaje del tiempo histórico en la Educación Primaria. Cuadernos Cedes, 82 (30). Barcelona, 281-309.

Paniagua G, M. N. (2013). Neurodidáctica: una nueva forma de hacer educación. Fides Et Ratio, 6, 72-77.

Rodríguez Palmero, Ma . L. (2004). La teoría del aprendizaje significativo. First International Conference on Concept Mapping. A. J. Cañas, J. D. Novak, F. M. González, Eds. Pamplona, Spain 
Rodríguez Palmero, Ma . L. (2011). La teoría del aprendizaje significativo: una revisión aplicable a la escuela actual. Revista Electrònica d'Investigació i Innovació Educativa i Socioeducativa, $3(1), 29-50$.

Santacana, J. y Hernández, X. (1999). Enseñanza de la arqueología y la prehistoria. Barcelona, Milenio.

Sousa, D. (2017). Neurociencia educativa. Mente, cerebro y educación. Madrid, Narcea

Stamm, J. (2018). Neurociencia infantil. El desarrollo de la mente y el poder del cerebro de 0 a 6 años. Madrid, Narcea.

Stenhouse, L (1984). Investigación y desarrollo del currículo. Madrid: Morata

Stenhouse, L. (1987). La investigación como base de la enseñanza. Madrid: Morata

Suárez, M.A. (2011). El Parque de la Prehistoria de Teverga: una mirada educativa entre lo potencial y lo posible, Her\&Mus, heritage \& museography, 7, 66-79.

Tate, M (2017). Cómo estimular el cerebro. En Sousa, D. Neurociencia educativa. Mente, cerebro y educación (pp.159-164). Madrid, Narcea 